Technological University Dublin

DÜBLIN

ARROW@TU Dublin

2012-6

\title{
Optimized Monopole and Dipole Antennas for UWB Asset Tag Location Systems
}

Antoine Dumoulin

Technological University Dublin

Matthias John

Technological University Dublin, matthias.john@tudublin.ie

Max Ammann

Technological University Dublin, max.ammann@tudublin.ie

See next page for additional authors

Follow this and additional works at: https://arrow.tudublin.ie/ahfrcart

Part of the Systems and Communications Commons

\section{Recommended Citation}

Dumoulin, A., et al. (2012) Optimized Monopole and Dipole Antennas for UWB Asset Tag Location Systems, Transactions on Antennas \& Propagation, IEEE, vol. 60, no. 6, pp. 2896-2904. DOI:10.1109/ TAP.2012.2194686

This Article is brought to you for free and open access by the Antenna \& High Frequency Research Centre at ARROW@TU Dublin. It has been accepted for inclusion in Articles by an authorized administrator of ARROW@TU

Dublin. For more information, please contact

arrow.admin@tudublin.ie, aisling.coyne@tudublin.ie, gerard.connolly@tudublin.ie.

Funder: SFI 
Authors

Antoine Dumoulin, Matthias John, Max Ammann, and Patrick McEvoy

This article is available at ARROW@TU Dublin: https://arrow.tudublin.ie/ahfrcart/40 


\title{
Optimized Monopole and Dipole Antennas for UWB Asset Tag Location Systems
}

\author{
Antoine Dumoulin, Member, IEEE, Matthias John, Member, IEEE, \\ Max J. Ammann, Senior Member, IEEE and Patrick McEvoy, Member, IEEE
}

\begin{abstract}
Miniaturized monopole and dipole antenna designs are reported with performances optimized for Ultra Wideband pulsed radio applications. The geometries are created using Bézier spline shapes, which have been refined with a genetic algorithm to simultaneously take account of both frequency- and time-domain criteria.

Time-domain measurements of ultra wideband antennas with uniformly distributed energy across the full 3.1 - 10.6 GHz mask are reported for the first time and validate a new approach to minimization of pulse dispersion effects in the antenna designs.
\end{abstract}

Index Terms - optimization methods, ultra wideband antennas, dipole antennas, time domain measurements.

\section{INTRODUCTION}

$\mathrm{T}$ HE IEEE 802.15.4a standard for Ultra Wideband (UWB) $(3.1-10.6 \mathrm{GHz})$ low-power short range communication devices also supports proximity ranging, or localization between UWB pulse-emitting devices, with centimeterprecision. Picosecond-scale pulses can produce a $7.5 \mathrm{GHz}$ bandwidth with uniform power levels and near-vertical slopes in the envelope edges for efficient use of the spectrum mask. Real Time Location Systems (RTLS) seek to exploit on the low distortion of the pulses to minimize detection errors and to extend the application coverage area.

Antenna designs for UWB Orthogonal Frequency Division Multiplexing (OFDM) devices have conventionally used optimization routines [1] that simply respond to frequencydomain parameters such as matched impedance bandwidth and radiated gain patterns. However, geometric iterations with this design method can lead to solutions with inherent non-linear phase characteristics that can adversely impact pulsed signals. Ergonomically packaged sensors usually require internal antennas and it is important that candidate designs with significant pulse-dispersion are avoided.

This paper presents two new planar printed antenna designs;

Manuscript received September 21 2011; revised December 11, 2011; accepted January 21, 2012. Date of publication Month XX, 2012; date of current version Month XX, 2012. The work was funded in part by Science Foundation Ireland grants 09/IN.1/I2652 and 10/CE/I1853 and a DIT Research Scholarship. A. Dumoulin, M. J. Ammann and P. McEvoy are with the Antenna and High Frequency Research Centre, Dublin Institute of Technology, Dublin 8, Ireland (e-mail: ammann@ieee.org). M. John is with the Telecommunication Research Centre (CTVR), Dublin Institute of Technology, Dublin 8, Ireland.

Colour versions of one or more of the figures in this paper are available online at http://ieeexplore.ieee.org.

Digital Object Identifier $\mathbf{X X X X}$ an unbalanced-fed monopole and a balance-fed dipole that are optimized for simultaneous high-performances in the frequency- and time-domains. The proposed antennas feature Bézier splines and are on low-cost FR4 substrates for integration with small, ultra wideband pulsed-signal radios.

The approach introduces the use of a Square Root Raised Cosine (SRRC) pulse, which contrasts with conventional Gaussian pulses, for an improved fit to the 802.15.4a power spectrum mask limits. The extremely broad bandwidth of the energy profile is used to qualify filtering by the antenna in terms of a fidelity factor (FF). The time-domain optimization uses a FF within the multi-objective goals of a genetic algorithm (GA), thereby minimizing dispersive properties.

A key aspect of validating the design approach includes the measurement of the SRRC pulse with a full UWB bandwidth through an antenna, reported here for the first time, using an arbitrary waveform generator. The measured frequency domain performances in terms of gain and matched bandwidths are also considered.

The optimization method and the design features produce novel miniaturized high-performance antennas that are suited to UWB asset tracking applications.

\section{EXCITATION PULSES}

UWB antenna literature predominantly reports Gaussian and Rayleigh pulses, and their derivatives, for antenna excitation [2]-[5]. Their characteristics include very low level side lobes in the frequency-domain and fast energy damping in the time-domain. However, the energy distribution across the frequency range is typically bell-shaped and antennas that are optimized with these pulse profiles do not have the bandwidth for an efficient fit to the 802.15.4a spectrum mask profile [4], [6], [7]. While a series of modulated Gaussian pulses can be sequenced [8] to cover the entire UWB mask, the approach increases the duration of the excitation pulse. It extends antenna simulation run-times and adds complexity to the time-domain analysis of the models.

Alternatively, an SRRC pulse overcomes the limitations. The analytical form of the SRRC pulse is shown in Eq.1 [9].

$$
F_{S R R C}(t)=\left[\frac{2 \beta}{\pi \sqrt{T_{S}}} \times \frac{\left.\cos \left[(1+\beta) \pi \frac{t}{T_{S}}\right]+\frac{\sin \left[(1-\beta) \pi \frac{t}{T_{S}}\right]}{4 \beta \frac{t}{T_{S}}}\right]}{\left[1-\left(4 \beta \frac{t}{T_{S}}\right)^{2}\right]}\right]
$$

where $t$ is the time, $T_{S}=1 / R_{S}, R_{S}$ being the symbol rate and $\beta$ is a dimensionless roll-off factor for bandwidth control. 


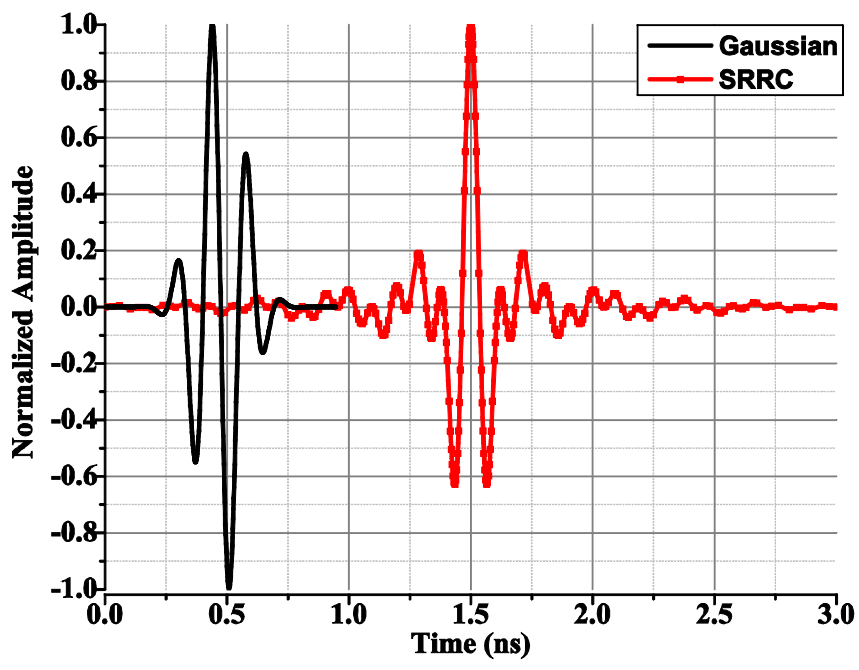

Fig. 1 Modulated Gaussian and Square Root Raised Cosine pulses with respective peak values normalized to unity

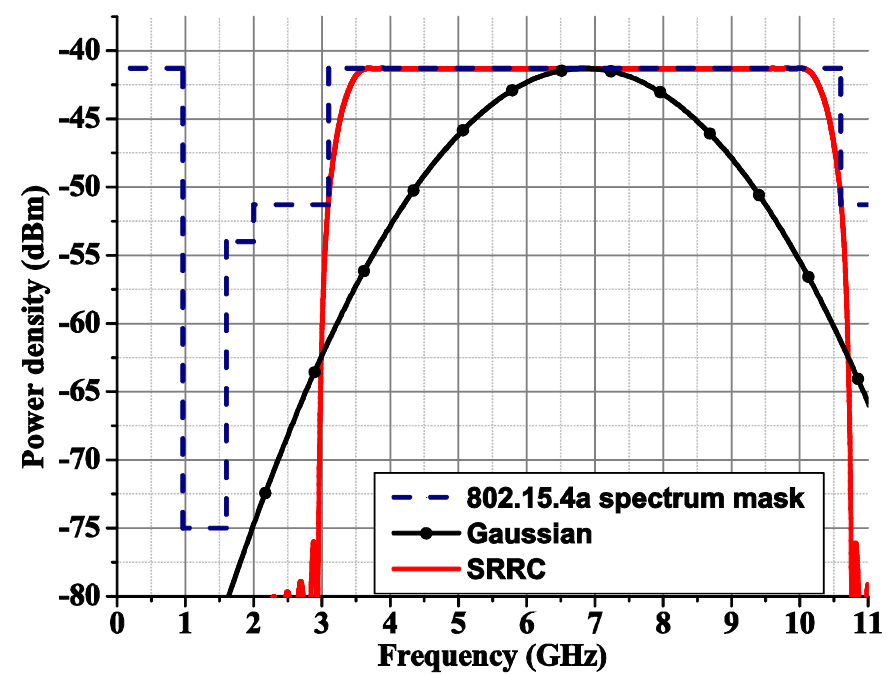

Fig. 2 802.15.4a indoor use spectrum mask with power spectrum density of Gaussian and SRRC pulses; respective peak values normalized to $-41.3 \mathrm{dBm}$

Fig. 1 shows an amplitude modulated Gaussian pulse and an amplitude modulated SRRC pulse (roll-off factor of 0.1 and symbol rate of $\left.7.1 \times 10^{9}\right)$; each centered at $6.85 \mathrm{GHz}$ and with the respective peak values normalized to unity. Fig. 2 shows the corresponding Power Spectrum Density (PSD) plots with the 802.11.4a UWB indoor use spectrum mask.

The evenly contoured power spectrum of the SRRC pulse, compared to that of the Gaussian pulse, conveys a broader bandwidth at a higher energy level. The design goal is to create antennas that can support pulses of increased bandwidths across the spectrum profile of the SRRC.

\section{ANTENNA GEOMETRIES}

Canonically shaped UWB antennas [10] have been analyzed for bandwidth properties but their simple geometric features constrain ultra wideband pulse performances. In order to solve the limitation posed by common shapes, Bézier splines have been used to generate contoured radiator and ground plane shapes, with an infinite number of configurations
[11]. The two types of antennas used in this study were optimized for wide-band performance and pulse fidelity. Gaussian Monopole (GM) in Fig. 3 and Gaussian Dipole $(G D)$ in Fig. 4 were optimized with a modulated Gaussian pulse. SRRC Monopole (SM) in Fig. 5 and SRRC Dipole (SD) in Fig. 6 were optimized using the modulated SRRC pulse shown in Fig. 1. In order to convey the bandwidth of the SRRC UWB pulse, both the radiator and ground plane were optimized. Monopoles $G M$ and $S M$ are microstrip-fed planar monopoles with an optimized geometry using a mirrored spline for the radiator (4 points) and the ground plane (4 points).

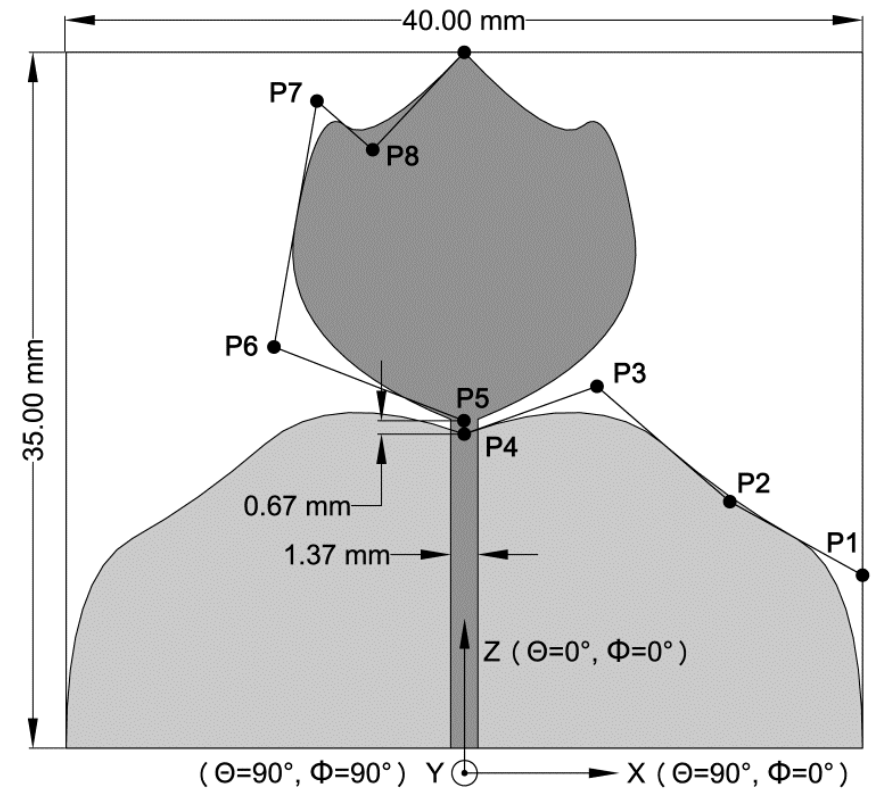

Fig. 3 Gaussian Monopole (GM) geometry and coordinate system

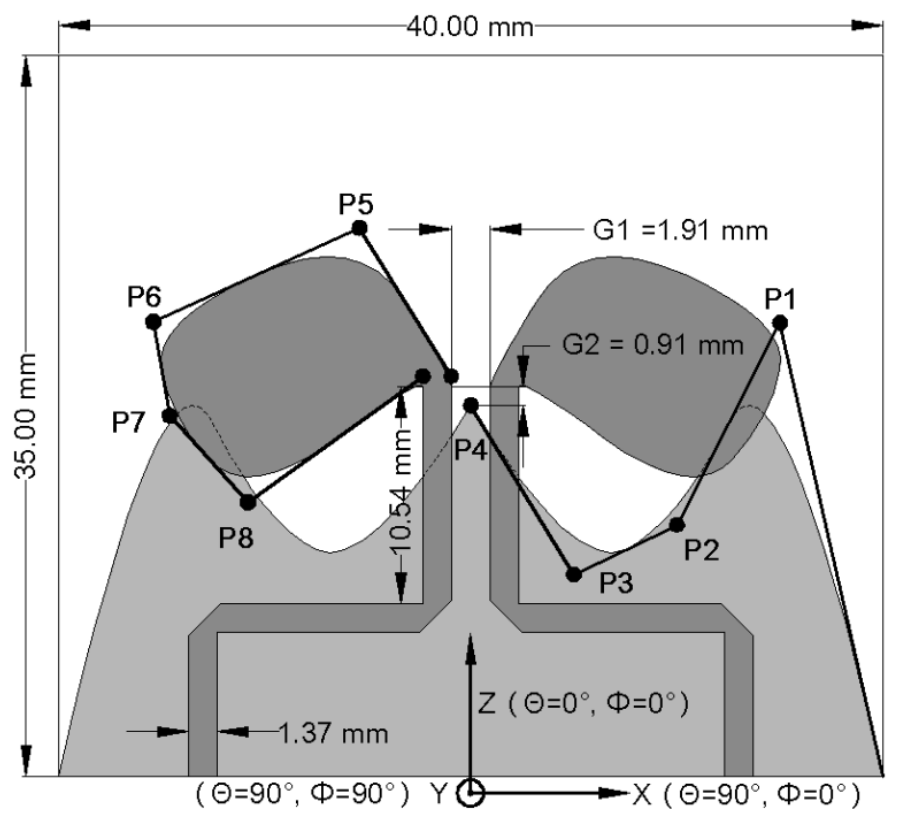

Fig. 4 Gaussian Dipole (GD) geometry and coordinate system 


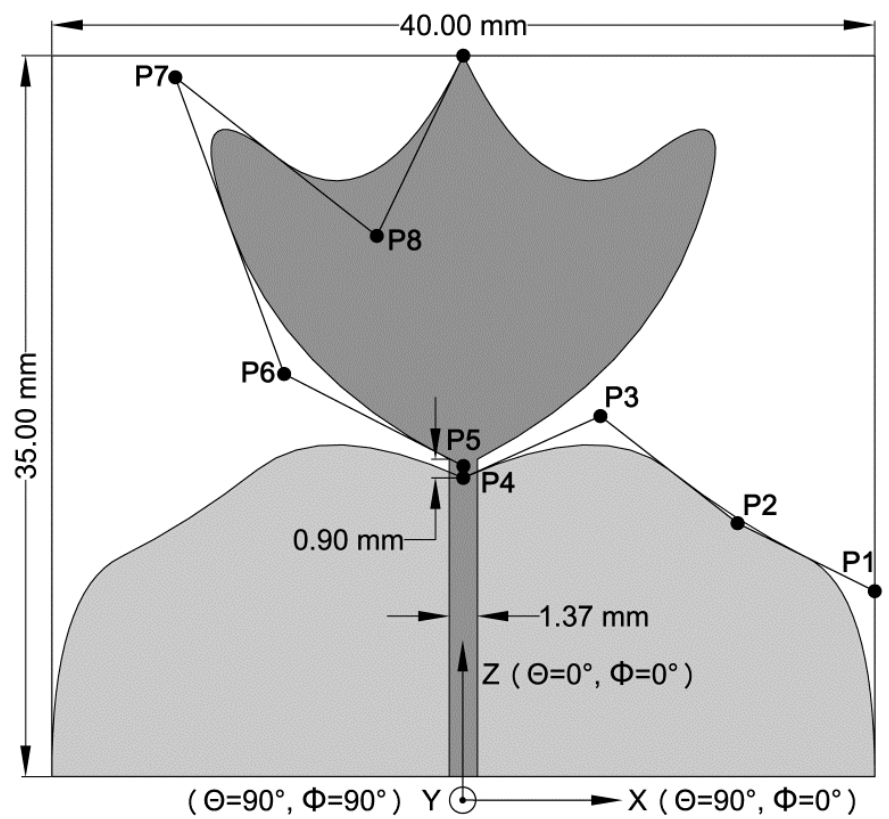

Fig. 5 SRRC Monopole (SM) geometry and coordinate system

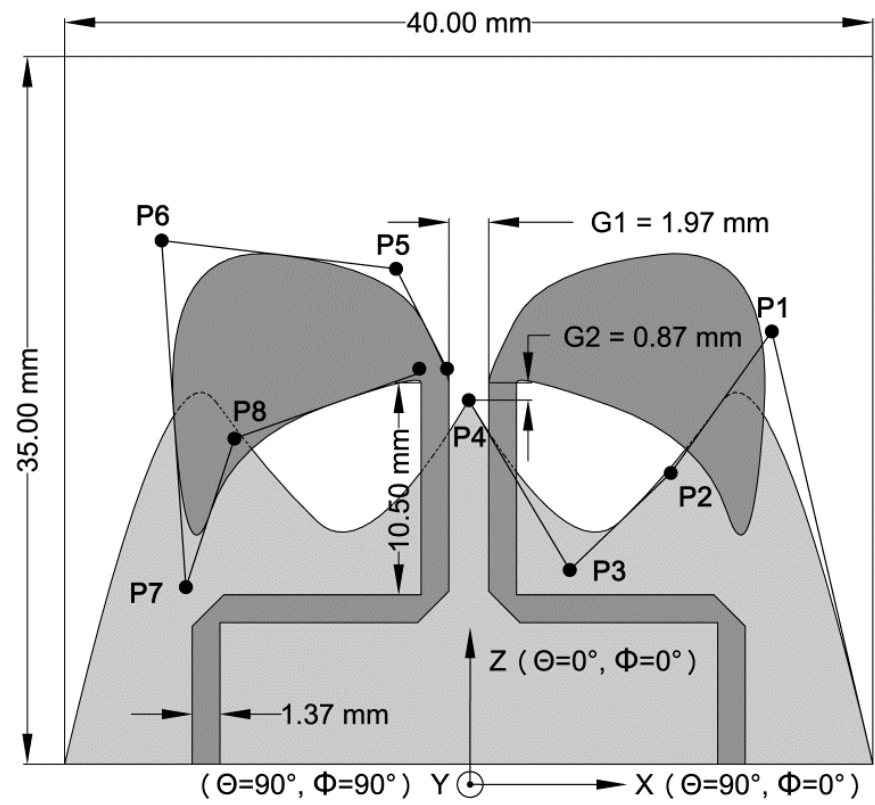

Fig. 6 SRRC Dipole (SD) geometry and coordinate system

Connection to the differential output of an IC chip source necessitates a balun for monopole antenna, which can introduce dispersive effects, or alternatively to use a balanced dual feed dipole antenna.

Antennas $G D$ and $S D$ are planar dipole-like with a balanced microstrip feed arrangement which requires a small ground plane. They consist of two identical dipole arms (defined by 4 spline points) fed with $50 \Omega$ dual mitered microstrip lines and a mirrored splined ground plane (4 points). For both the monopole and dipole antennas, the spline points for the radiators have two degrees of freedom ( $x$ - and $z$-axis), while the spline points for the ground plane are restricted to one degree of freedom (z-axis).

The antennas were fabricated on a $40 \times 40 \times 0.7 \mathrm{~mm}$ FR4 double-sided laminate with a dielectric constant $\varepsilon_{r}=4.3$ and loss tangent $=0.02$. The antennas were initially performanceoptimized when configured with the system circuitry in the simulation model; therefore without SMA connectors. However, for measurement validation, additional comparison simulation models with SMA connectors were analyzed.

\section{DESIGN OptIMIZATION}

Antenna simulations in CST's Microwave Studio were optimized with the MATLAB Genetic Algorithm. By assigning dimensional limits to 12 optimization parameters, the GA evolves towards a preferred performance goal. The dimensional constraints are defined in millimeters as:

Monopole

- $P_{1,2,3,4}(z)=5<z<2$

- $P_{5}(z)=P_{4}<z<\left(P_{4}+5\right)$

- $P_{6,7,8}(x)=0<x<20$

- $P_{6,7,8}(z)=P_{5}<z<40$
Dipole

- $P_{1,2,3,4}(z)=5<z<26$

- $P_{5}(x)=0<x<4$

- $P_{5}(z)=P_{4}<z<40$

- $P_{6,7,8}(x)=10<x<20$

- $P_{6,7,8}(z)=P_{5}<z<40$

- $G_{1}=1<x<5$

- $G_{2}=0<z<5$

For time-domain optimization, field-probes were located $30 \mathrm{~cm}$ from the monopoles in the azimuth plane. Simulation time was halved by using field symmetry in the H-plane and by limiting probes to $\left[\theta=90^{\circ}, 90^{\circ} \leq \varphi \leq 270^{\circ}\right.$ in $5^{\circ}$ steps]. In the case of the dipoles $G D$ and $S D$, the optimizer field probes were configured $\left[\varphi=90^{\circ},-90^{\circ} \leq \theta \leq 90^{\circ}\right.$ in $5^{\circ}$ steps $]$.

A weighted cost function was post-processed for the design iterations using Eq. $2 a$ and $2 b$.

$$
\begin{array}{ll}
\text { Cost }=-0.3 \times \frac{\Gamma}{\alpha}-0.7 \times \frac{\sum F F}{\gamma}, & \text { for } \Gamma<\alpha \\
\text { Cost }=-0.3-\left(0.7 \times \frac{\sum F F}{\gamma}\right), & \text { for } \Gamma \geq \alpha
\end{array}
$$

where $\Gamma$ is the least matched magnitude in the $S_{11}, \alpha$ is the $S_{11}$ match target, $F F$ is the fidelity factor [12] (field probes relative to the derivative of the excitation pulse) at each angle and $\gamma$ is the number of FF values. The empirically selected coefficient weightings were chosen to provide a balance between optimization time and accuracy.

Additionally, a subroutine was used to skip simulations of non-realistic structures or re-simulation of identical structures [13], more of which occur as the algorithm converges on the optimum range of possible solutions. These significantly reduce the optimization time with minimum effect on the GA behavior. The algorithm also uses an intermediate crossover, the roulette wheel selection, and stops after 30 population iterations (maximum antenna simulations $\leq 8085$ ).

Fig. 7 represents the two objectives (X-Y axis) in terms of the sequence of simulations used to optimize Monopole GM. The first 1650 iterations indicate that the GA considered a reasonably broad range of random possibilities to avoid overlooking solutions. In the subsequent iterations, the GA progressively refines the remaining parameter options towards the optimum values, which are darker in color. 


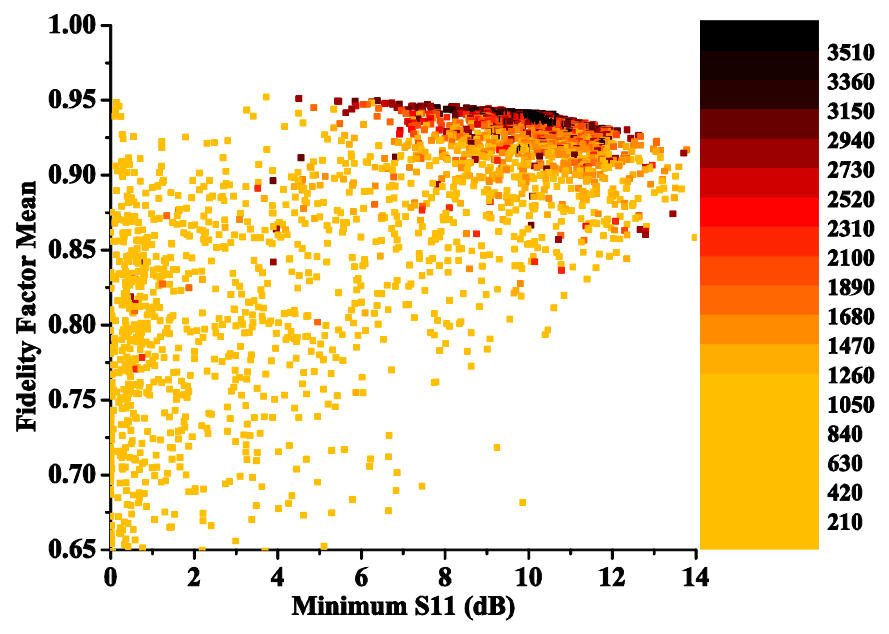

Fig. 7 Optimization goal in terms of iteration number

\section{FREQUENCY-DOMAIN RESULTS}

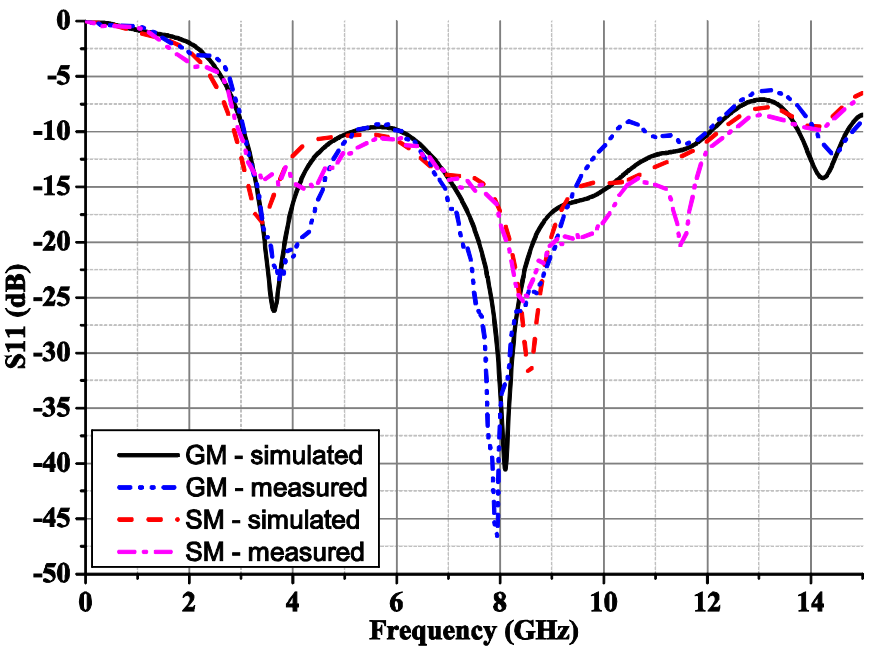

Fig. $8 \mathrm{~S}_{11}$ for Monopole GM and Monopole SM

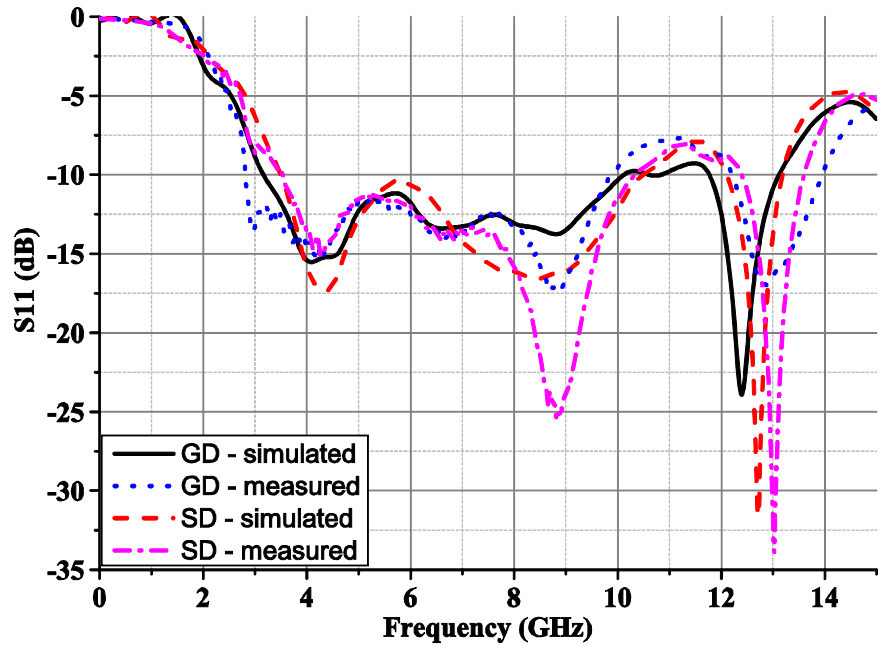

Fig. $9 \mathrm{~S}_{11}$ for Dipole $G D$ and Dipole $S D$

Measurements were made using a Rohde \& Schwarz ZVA 24 network analyzer. The simulated and measured $\mathrm{S}_{11}$ for Monopole GM and Monopole SM are illustrated in Fig. 8. Both monopoles exhibit a $10 \mathrm{~dB}$ return loss for $3.06-12.21 \mathrm{GHz}$ and $2.97-12.22 \mathrm{GHz}$, respectively. Fig. 9 shows the measured $\mathrm{S}_{11}$ for Dipole $G D$ and Dipole $S D$. The dipoles exhibit an $8 \mathrm{~dB}$ return loss across $2.75-10.6 \mathrm{GHz}$ and $2.9-13.8 \mathrm{GHz}$, respectively. The antennas have good impedance matching for the $3.1-10.7 \mathrm{GHz}$ band, with good agreement between simulations and measurements.

The monopole radiation patterns were measured in the $\mathrm{H}$-plane (X-Y plane) from $3-11 \mathrm{GHz}$ to evaluate the stability of the radiation pattern. The measured co-polar gain is plotted against frequency and azimuth angle. The radiation patterns for Monopole GM and Monopole SM are shown in Fig. 10 and Fig. 11, respectively. The gain values are good across $3.1-10.6 \mathrm{GHz}$ with an averages of $0.75 \mathrm{dBi}($ std. dev. $=2.29)$ for Monopole GM and $0.8 \mathrm{dBi} \quad$ (std. dev. =2.23) for Monopole SM. The respective maximum gains are $4.53 \mathrm{dBi}$ $\left(\theta, \varphi=90^{\circ}, 40^{\circ}\right)$ at $8.27 \mathrm{GHz}$ and $4.51 \mathrm{dBi}\left(\theta, \varphi=90^{\circ}, 25^{\circ}\right)$ at $8.93 \mathrm{GHz}$.

The dipole radiation patterns were similarly measured in the H-plane (Z-Y plane). Fig. 12 and Fig. 13 show good stability in the radiation pattern across $3.1-10.6 \mathrm{GHz}$. The dipoles achieve an average realized gain of $-0.27 \mathrm{dBi}($ std. dev. $=2.45)$ and $0.8 \mathrm{dBi}($ std. dev. $=2.47$ ) respectively. Dipole $G D$ has a maximum gain of $6.4 \mathrm{dBi}\left(\theta, \varphi=90^{\circ}, 55^{\circ}\right)$ at $8.88 \mathrm{GHz}$, while Dipole $S D$ has the maximum gain of $6.87 \mathrm{dBi}\left(\theta, \varphi=90^{\circ}, 60^{\circ}\right)$ at $9.4 \mathrm{GHz}$.

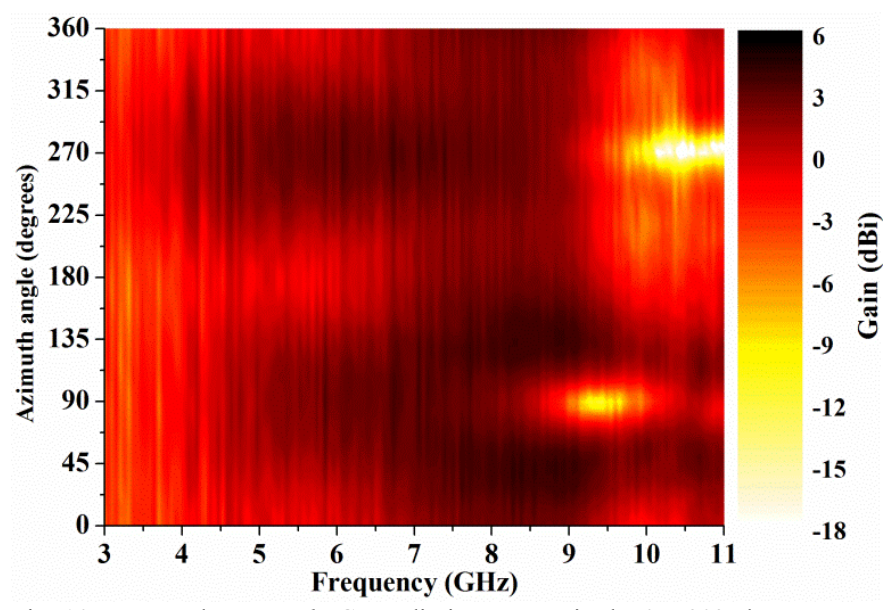

Fig. 10 Measured Monopole $G M$ radiation pattern in the $\theta=90^{\circ}$ plane

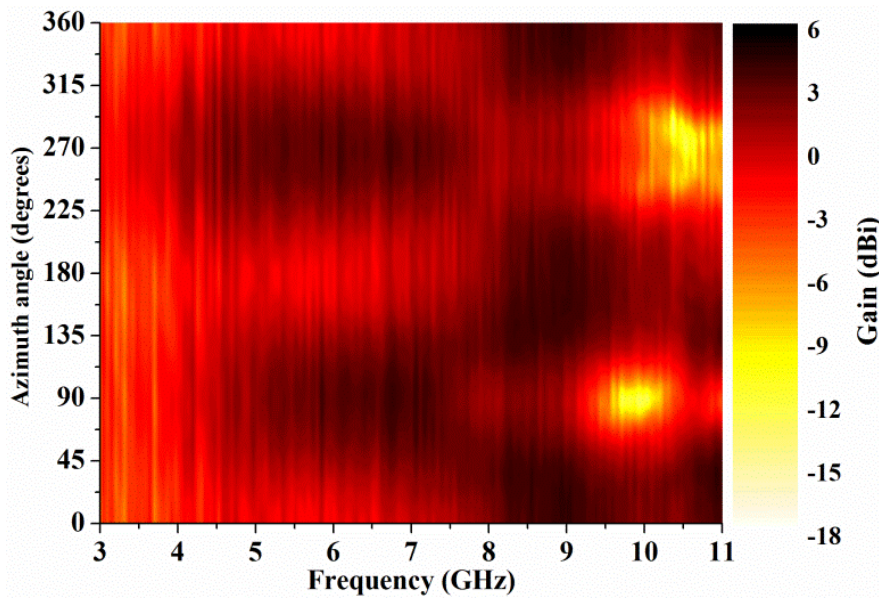

Fig. 11 Measured Monopole $S M$ radiation pattern in the $\theta=90^{\circ}$ plane 


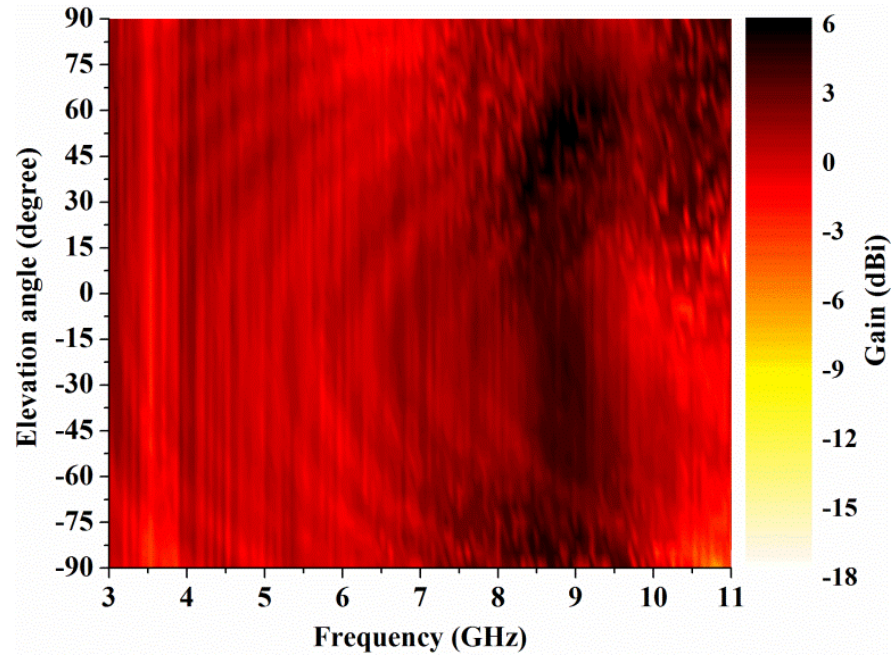

Fig. 12 Measured Dipole $G M$ radiation pattern in the $\varphi=90^{\circ}$ plane

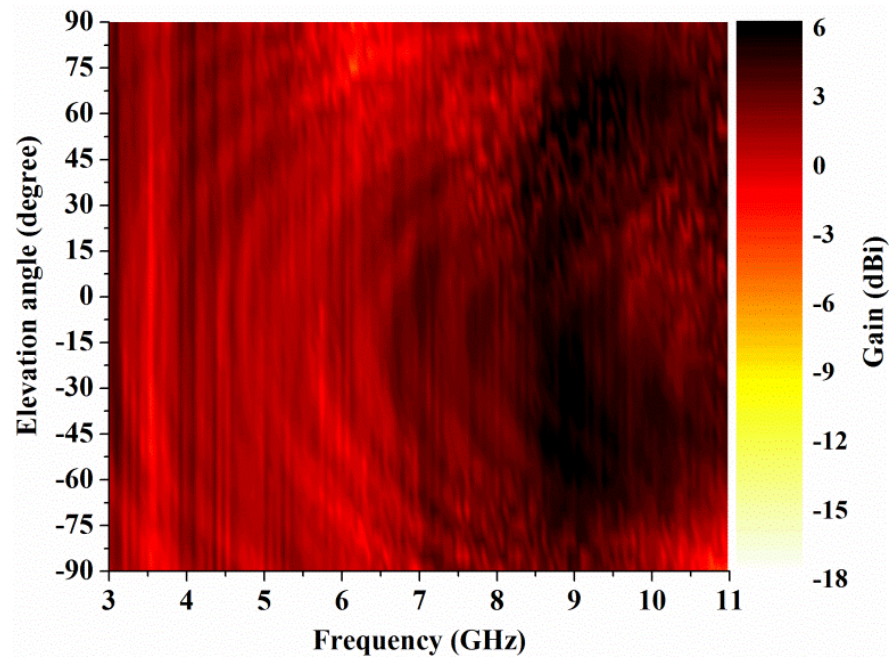

Fig. 13 Measured Dipole $S D$ radiation pattern in the $\varphi=90^{\circ}$ plane

\section{Simulated TIME-DOMAIN Results}

To quantify the time-domain performance of an antenna, or a system of two antennas, the most common metric is the FF. The factor is established by the maximum absolute value of the cross-correlation coefficient of the two normalized pulses. It is a useful measure of how much time-domain dispersion an antenna will add to a pulse being transmitted through it.

The antenna and its operating mode determine if the radiated pulse will be radically different to the source pulse. It has been shown that the transient response of an antenna in transmitting mode is proportional to the time derivative of the impulse response of the same antenna in receiving mode [14] - [16]. Researchers have demonstrated that radiation of UWB pulses involve fields that are time-delayed timederivatives of the signal currents from the various parts of the transmitting antenna [17]. Hence, the simulated radiated pulses have to be cross-correlated with the $1^{\text {st }}$-order derivative of the excitation pulse.

In order to compare the time-domain performance of the monopoles and dipoles, the antennas were fed with a modulated Gaussian pulse and a modulated SRRC UWB pulse with an improved fit with the FCC UWB indoor mask. Fig. 14 shows the FF polar plot for antennas fed with a modulated Gaussian pulse, while Fig. 15 shows the FF when using the SRRC pulse (see Fig. 1) as excitation pulse.

Inspection of Fig. 14 and Fig. 15 suggests that the FF values for simulated antennas excited with a modulated SRRC pulse are inferior to those fed with the modulated Gaussian pulse. A FF measurement for a wider bandwidth pulse is subject to more frequency dependent filtering effects from an antenna.

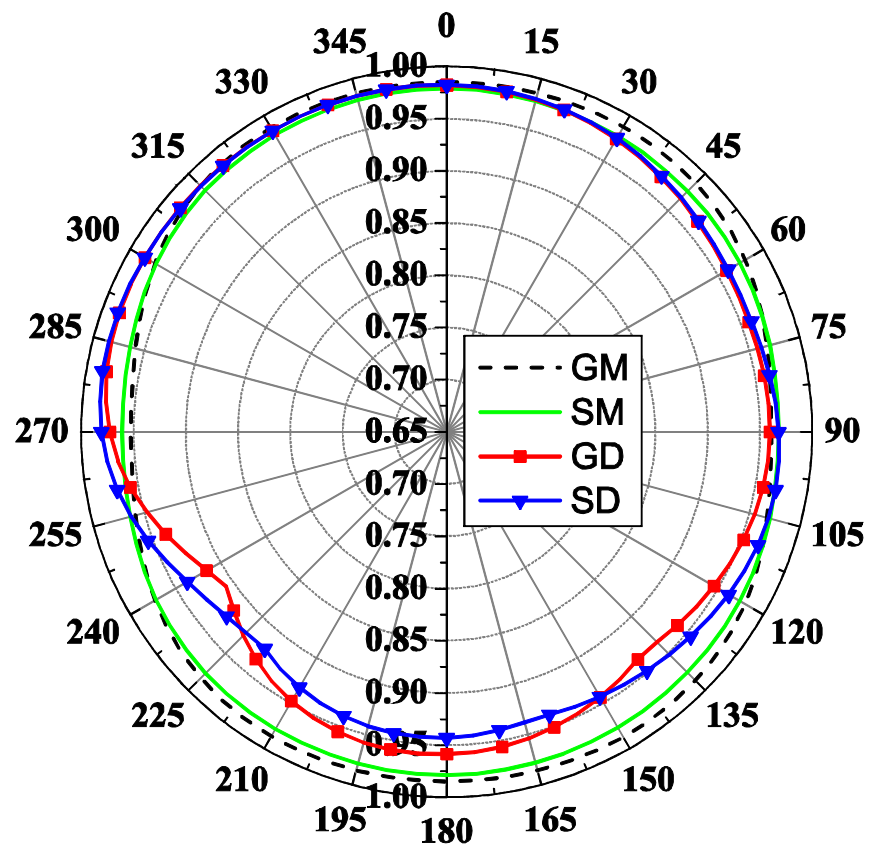

Fig. 14 Simulated FF for antennas fed by modulated Gaussian pulse

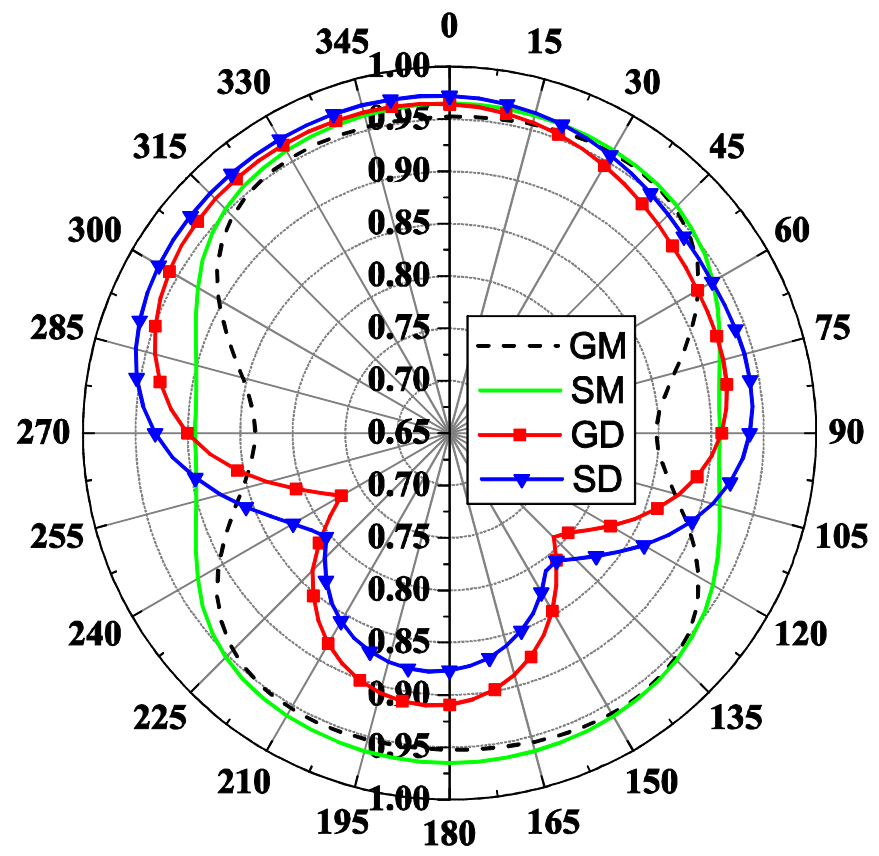

Fig. 15 Simulated FF for antennas fed by modulated SRRC pulse 
Table 1 FF percent values for antennas fed by Gaussian pulse

\begin{tabular}{|l|c|c|c|c|}
\hline & $\begin{array}{c}\text { Monopole } \\
\text { GM }\end{array}$ & $\begin{array}{c}\text { Monopole } \\
\text { SM }\end{array}$ & $\begin{array}{c}\text { Dipole } \\
\text { GD }\end{array}$ & $\begin{array}{c}\text { Dipole } \\
\text { SD }\end{array}$ \\
\hline Mean & 97.801 & 97.395 & 97.501 & 97.666 \\
\hline Min & 95.237 & 96.084 & 95.762 & 96.019 \\
\hline Max & 98.776 & 97.888 & 98.358 & 98.489 \\
\hline
\end{tabular}

Table $2 \mathrm{FF}$ percent values for antennas fed by SRRC pulse

\begin{tabular}{|l|c|c|c|c|}
\hline & $\begin{array}{c}\text { Monopole } \\
\text { GM }\end{array}$ & $\begin{array}{c}\text { Monopole } \\
\text { SM }\end{array}$ & $\begin{array}{c}\text { Dipole } \\
\text { GD }\end{array}$ & $\begin{array}{c}\text { Dipole } \\
\text { SD }\end{array}$ \\
\hline Mean & 93.557 & 95.598 & 94.542 & 95.884 \\
\hline Min & 83.538 & 89.323 & 90.029 & 93.092 \\
\hline Max & 95.948 & 96.498 & 96.765 & 97.416 \\
\hline
\end{tabular}

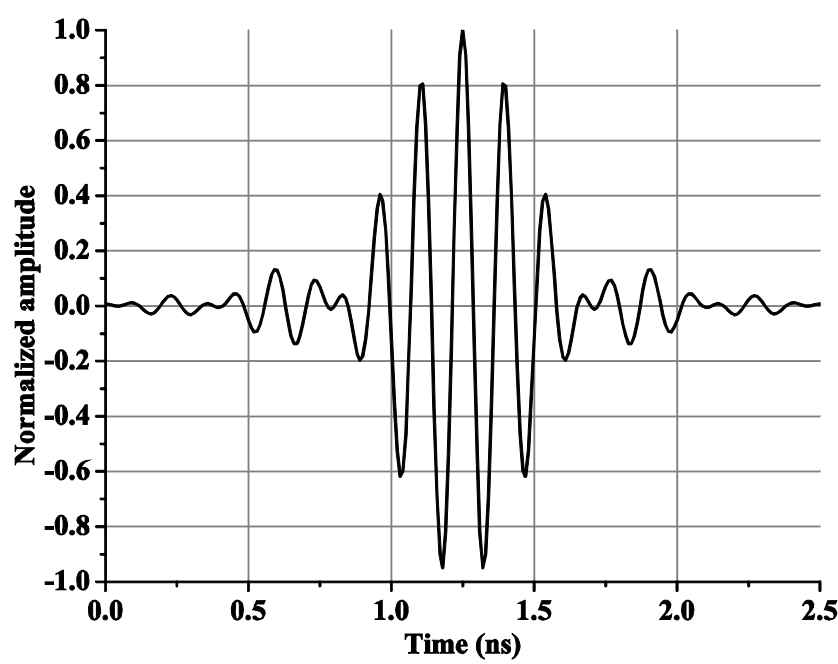

Fig. 16 SRRC pulse: $2.5 \mathrm{GHz}$ bandwidth, modulated at $6.85 \mathrm{GHz}$, with the peak value normalized to unity

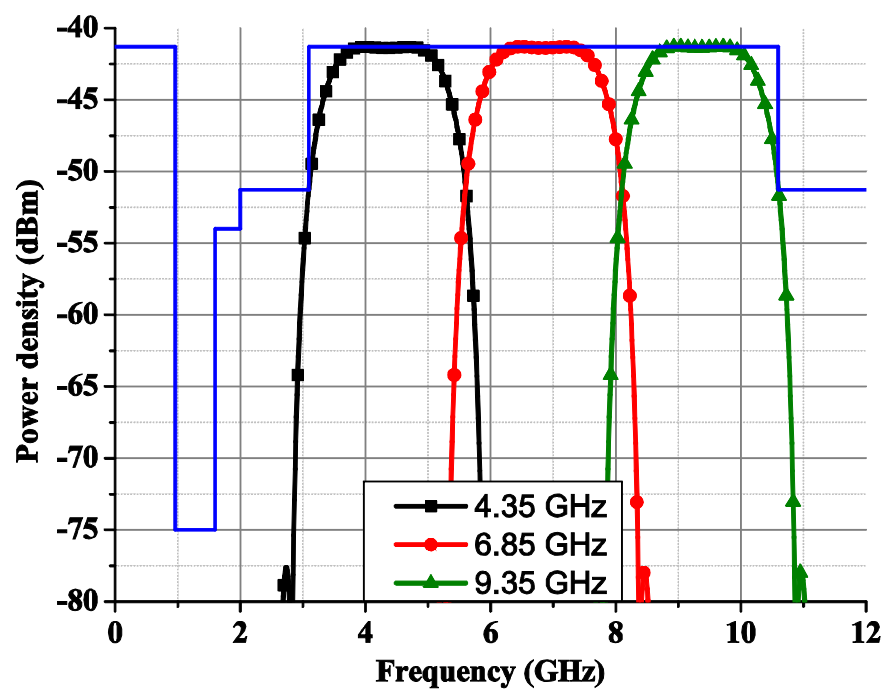

Fig. 17 Spectrum Power Density for $2.5 \mathrm{GHz}$ bandwidth SRRC pulse at various center frequencies

Table 1 and Table 2 indicate the FF performance range for the field probe location angles specified in section IV. Table 1 indicates that the TD characteristic for Monopole GM outperformed the Monopole SM, while the contrary outcome is displayed in Table 2, even though the Monopole SM was optimized with SRRC pulse. To explore the apparent contradiction, each antenna was stimulated with three narrower-band SRRC excitation pulses $(\beta=0.5$ and
$\mathrm{R}_{\mathrm{S}}=1.95 \times 10^{9}, 2.5 \mathrm{GHz}$ bandwidth at $-10 \mathrm{~dB}$ ) with various center frequencies across the UWB band (see Fig. 16 and Fig. 17).

Table 3 Fidelity Factor (\%) for narrow band SRRC pulses

\begin{tabular}{|l|c|c|c|}
\hline & $4.35 \mathrm{GHz}$ & $6.85 \mathrm{GHz}$ & $9.35 \mathrm{GHz}$ \\
\hline Monopole GM & 97.563 & 99.647 & 97.329 \\
\hline Monopole SM & 98.016 & 99.270 & 99.293 \\
\hline
\end{tabular}

Table 3 illustrates that an antenna optimized with a SRRC pulse, or with a pulse having an even energy level across the frequency range, achieved a better overall TD performance compared with the Gaussian pulse optimization. However Monopole GM achieved better performance than Monopole SM for the pulse centered at $6.85 \mathrm{GHz}$. Again this result is due to the fact that this antenna has been optimized for a Gaussian excitation pulse having its peak energy centered at $6.85 \mathrm{GHz}$.

The results indicate that the type of excitation pulse used in optimization governs how the optimization algorithm will influence an antenna's geometric features. It is clear that Monopole SM clearly outperforms Monopole GM in the band where the Gaussian pulse energy distribution is inferior to the energy distribution from the SRRC pulse. Accordingly, SRRC optimized antennas have a better overall TD performance than Gaussian optimized antennas.

\section{TIME-Domain MEASUREMENT SETUP}

Fig. 18 is a schematic outline of the measurement setup for the dipole antenna configuration. A Tektronix AWG7122C Arbitrary Waveform Generator (AWG) synthesizes an SRRC UWB pulse from a sampled waveform of points generated by the SRRC equation. The AWG has a peak voltage limitation, so a Picosecond Pulse Labs wideband amplifier (Model 5865) was used to boost the output. The antennas under test were connected to the amplifier via a rotary joint and mounted on a turntable. In the case of the balance-fed dipole antennas, an ultra wideband anti-phase power divider was employed [18] to produce the $180^{\circ}$ differential between the dipole elements.

The standard reference antenna should not add significant dispersion when receiving the incident pulse and any effects are eliminated by de-embedding [19]-[21]. A bore-sight directional tapered slot antenna was selected for the nondispersive TD performance in receive mode. The reference antenna was aligned $20 \mathrm{~cm}$ distant from the transmitting antenna and connected to an Agilent DSO81204A oscilloscope with a $12 \mathrm{GHz}$ sampling bandwidth. The $20 \mathrm{~cm}$ distance was selected to balance the need for far-field measurements with an adequate signal-to-noise ratio. Distances exceeding $50 \mathrm{~cm}$ proved too unreliable for comparative measurements of antennas that were examined during the measurement configuration. The amplifier output was $7 \mathrm{dBm}$ and the oscilloscope measurement noise floor was $-54 \mathrm{dBm}$. For the monopole setup, the antenna input power was $4.5 \mathrm{dBm}$ while the power at the receiving antenna port was circa $-25.7 \mathrm{dBm}$. For the dipole setup, the antenna input power was $0 \mathrm{dBm}$ while the power at the receiving antenna port was circa $-29.2 \mathrm{dBm}$. 


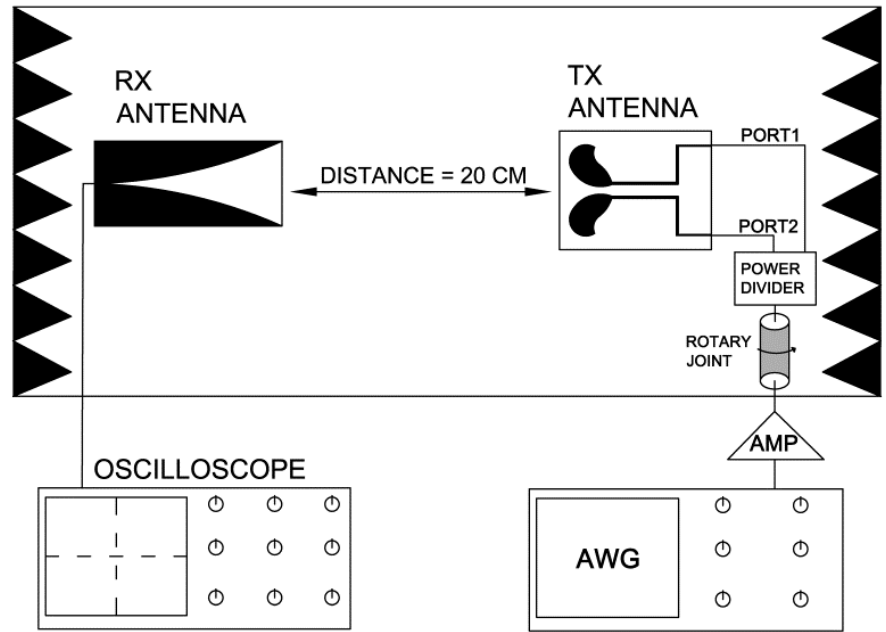

Fig. 18 TD measurement setup for dipole antennas

\section{PULSE EQUALIZATION}

The AWG generated UWB SRRC pulse is degraded by the transient response of the transmission network and this must be isolated to assess the antenna under test. Fig. 19 contrasts the input waveform in(t) to the AWG with the pulse out $(t)$ offered to the transmit antenna. An equalized pulse $i n^{\prime}(t)$ is derived numerically with Eq. (3).

$$
\operatorname{in}^{\prime}(t)=\operatorname{IFFT}[F F T(\operatorname{in}(t)) / F F T(\operatorname{out}(t))]
$$

where the Inverse Fast Fourier Transform (IFFT) and the Fast Fourier Transform (FFT) are used to manipulate the pulse in the frequency domain. Fig. 20 and Fig. 21 show the power spectrum densities and the modified pulse waveforms for $i^{\prime}(t)$ and out $^{\prime}(t)$, respectively. Fig. 21 shows that the equalized pulse out $^{\prime}(t)$ is similar to the basic SRRC waveform. Different equalization values are used for the dipole configuration.

\section{Measured Time-Domain Results}

The transmitting antenna was rotated through $15^{\circ}$ angle steps in the radiation plane. The oscilloscope was set to 64sweep averaging to mitigate spurious sampling artifacts in the measured waveforms. Separate equalization eliminated the dispersive effects due to the cables between the reference antenna and the oscilloscope. The resultant measurement data for the antenna under test was then equivalent to the simulated field-probe data.

The FF was computed by correlating the post-processed received pulse waveforms with the $1^{\text {st }}$ order derivative of the AWG output pulse out'(t). Fig. 22 and Fig. 23 show the measured FF polar plot for Monopole GM, Monopole SM, Dipole $G D$ and Dipole $S D$ alongside the simulated results from a modulated SRRC excitation pulse. Good agreement between simulation and measurement is achieved. Furthermore, the measurements validate that an antenna optimized for the full SRRC UWB pulse (Monopole SM and Dipole $S D$ ) has better time-domain performance for the full UWB range than an antenna optimized with a pulse that does not fit to the FCC UWB spectral mask (Monopole GM and Dipole GD).

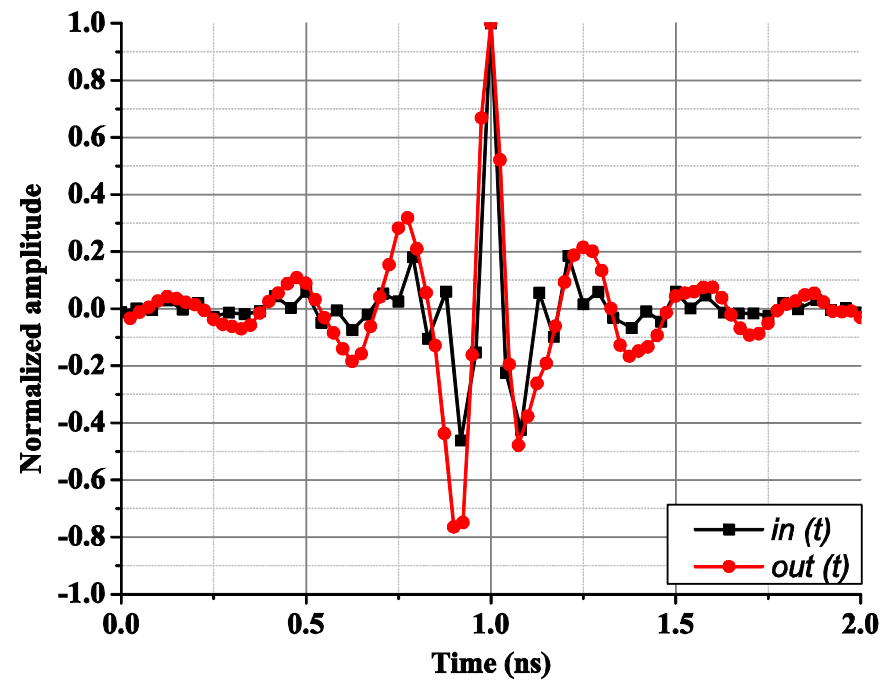

Fig. $19 \mathrm{in}(t)$ waveform to AWG and out $(t)$ pulse for Monopole SM setup

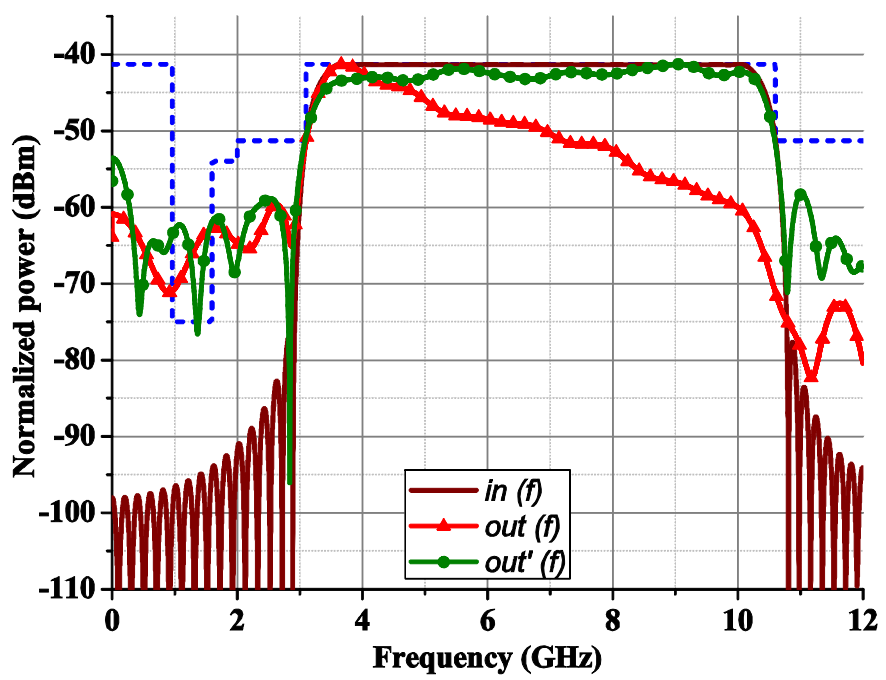

Fig. 20 input, output and output equalized pulse Power Spectrum Density for Monopole SM setup

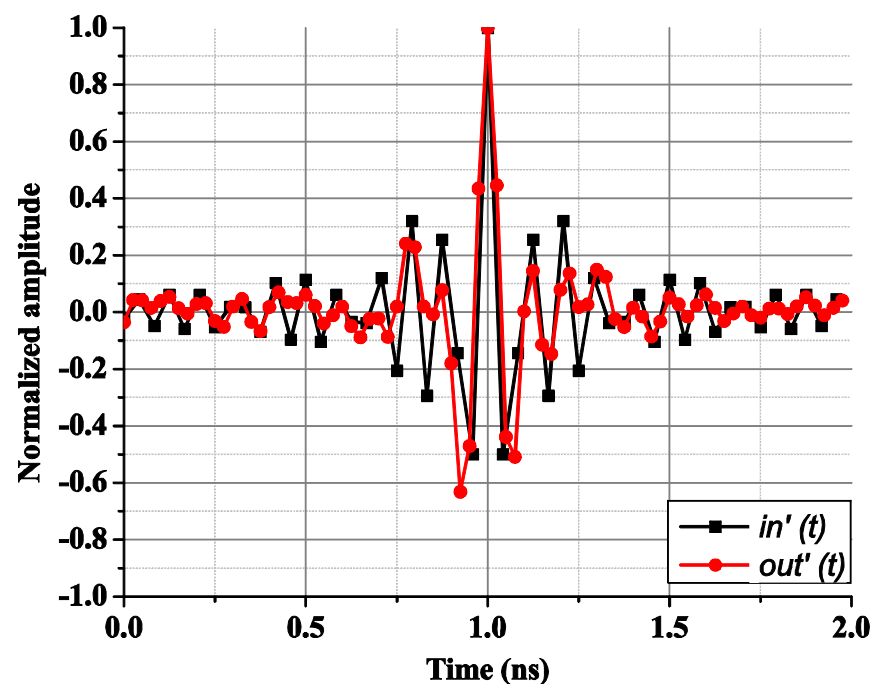

Fig. 21 Equalized AWG input and output pulses for Monopole SM setup 


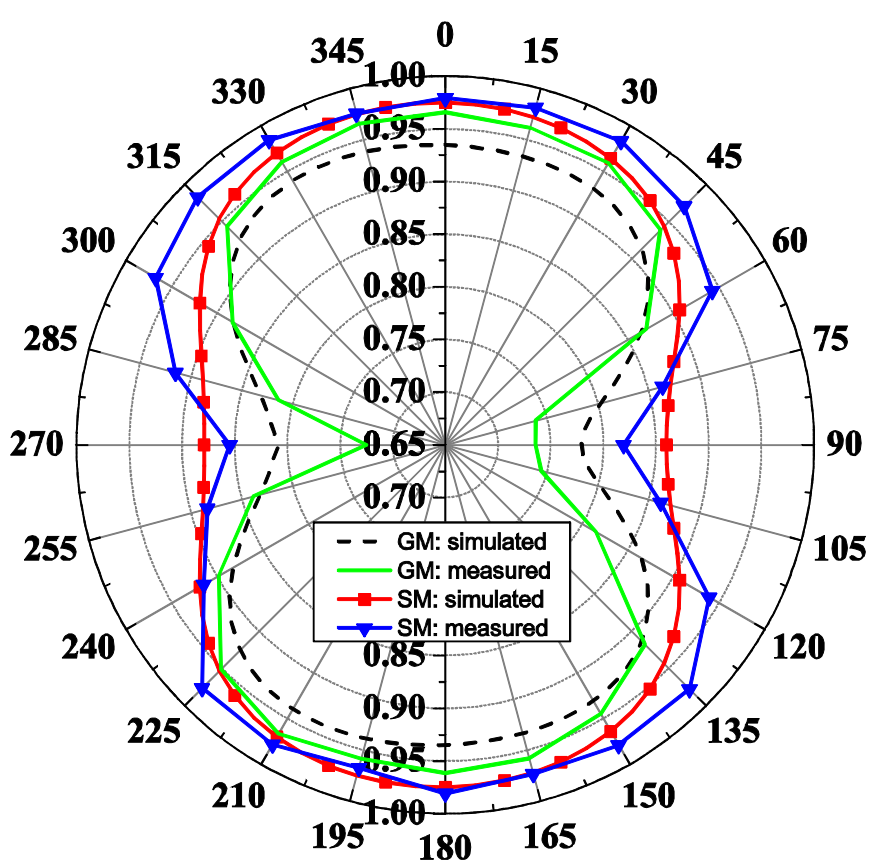

Fig. 22 Measured and simulated FF for an antenna system for Monopole GM and Monopole SM with an equalized modulated SRRC excitation pulse

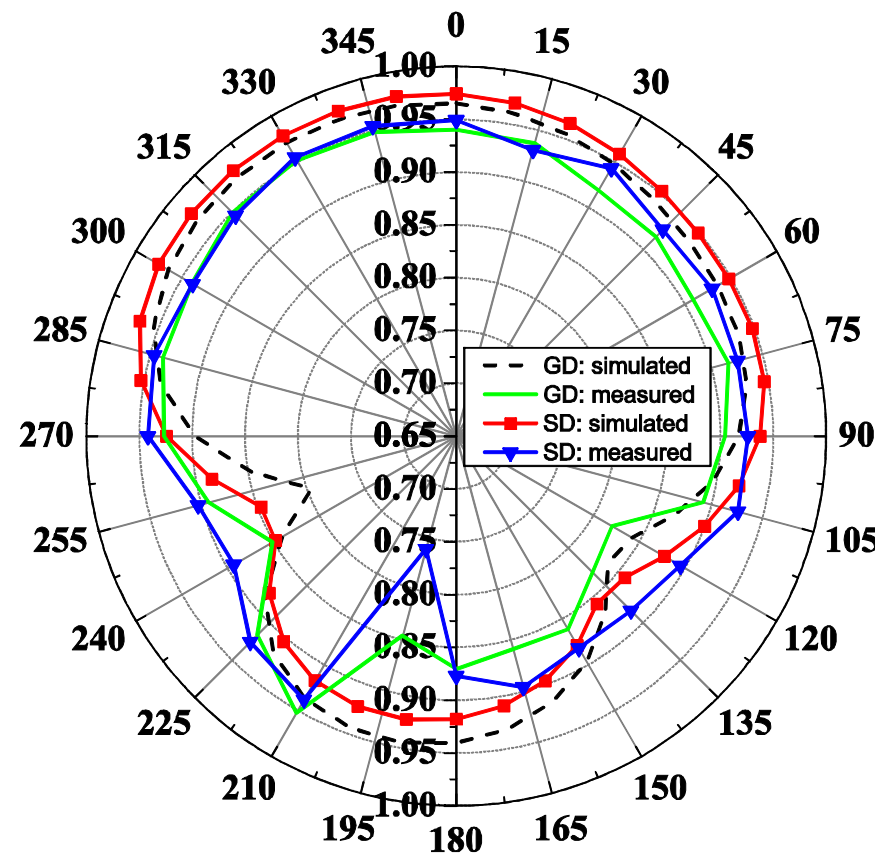

Fig. 23 Measured and simulated FF for an antenna system for Dipole GD and Dipole $S D$ with an equalized modulated SRRC excitation pulse

\section{CONCLUSION}

This paper demonstrates that the time-domain performance of an antenna can be optimized according to the type of UWB pulse to be used in the application device. Some novel highperformance monopole and dipole antennas are proposed as solutions for pulsed asset tag devices. Fidelity factors of $93.5 \%$ and better are proven suitable for the full $3.1-10.6 \mathrm{GHz}$ and have been validated for the first time with measurements.
Due to the complexity of the antenna geometry, extensive optimization has been carried out while a computation time saving method has been implemented. Frequency-domain measurement revealed that the optimized antennas achieved good matching and radiation pattern, throughout the full FCC 802.15.4a frequency range.

The TD performance of the antenna was further analyzed by assessing the antennas with pulses having a third of the FCC bandwidth. It demonstrates that SRRC optimized antennas have a better time-domain performance than Gaussian optimized antennas throughout the frequency range.

\section{ACKNOWLEDGMENT}

The authors would like to thank Liam Barry at the Radio and Optical Communications Laboratory, Dublin City University and Philip Perry at Pilot Photonics for access to pulse generation equipment.

The authors would also like to thank IMEX Instruments Ltd. and Tektronix for use of an Arbitrary Waveform Generator $7122 \mathrm{C}$ and Andrew Ellis, Jian Zhao and Naoise MacSuibhne at Tyndall National Research Institute, Cork, Ireland for use of equipment purchased under Science Foundation Ireland grant 06/IN/I969.

\section{REFERENCES}

[1] F. J. Villegas, T. Cwik, Y. Rahmat-Samii, and M. Manteghi, "A parallel Electromagnetic Genetic-Algorithm Optimization (EGO) application for patch antenna design," IEEE Trans. Antennas Propag., vol. 52, no. 9, pp. 2424-435, Sept. 2004.

[2] Z.-N. Chen, X. H. Wu., H. F. Li, N. Yang and M. Y. W. Chia, "Considerations for source pulses and antennas in UWB radio systems", IEEE Trans. Antennas Propag., vol. 52, no. 7, pp. 1739-1748, Jul. 2004.

[3] L. Lizzi, L. Manica, A. Massa, "Time-domain analysis for UWB antenna synthesis", in Proc. $39^{\text {th }}$ European Microw. Conf., Rome, 2009, pp. 93-96.

[4] Z.-N. Chen, X. Qing and M. Y. W. Chia, "UWB characteristics of disc cone antenna", in IEEE Int. Workshop on Antennas Technology, Marina Mandarin, Singapore, 2005, pp. 97-100.

[5] L. Guo, J. Liang, C. C. Chiau, X. Chen, C. G. Parini and J. Yu, "Performance of UWB disc monopoles in time-domain", Microw. Antennas and Propag., vol. 1, no. 4, pp. 955-959, Aug. 2007

[6] S. Chamaani, S. A. Mirtaheri and M. S. Abrishamian, "Improvement of time frequency-domain performance of antipodal Vivaldi antenna using multiobjective particle swarm optimization", IEEE Trans. Antennas Propag., vol. 59, no. 5, pp. 1738-1742, May 2011.

[7] P. Cerny and M. Mazanek, "Optimization of transient response radiation of printed ultra wideband dipole antennas", Radioengineering, vol. 16, no. 2, pp. 1-6, 2007.

[8] Part 15.4: Wireless Medium Access Control (MAC) and Physical Layer (PHY) Specifications for Low-Rate Wireless Personal Area Networks (WPANs), IEEE Standard 802.15.4a, 2007.

[9] X. Zhang, L. E. Larson and P. M. Asbeck "Design of linear RF outphasing power amplifiers", Artech house Inc., 2003, pp. 47

[10] X. H. Wu and Z.-N. Chen, "Comparison of planar dipole in UWB applications", IEEE Trans. Antennas Propag., vol. 53, no. 6, pp. 19731983, June 2005.

[11] M. John and M. J. Ammann, "Spline-based geometry for printed monopole antennas", Electronics Letters, vol. 43, no. 6, pp. 7-8, Mar. 2005.

[12] D. Lamensdorf and L. Susman, "Baseband-pulse-antenna techniques", IEEE Antennas and Propag. Mag., vol. 36, no. 1, pp. 20-30, Feb. 1994.

[13] R. L. Haupt and S. E. Haupt, "Practical genetic algorithms", WileyInterscience, 2004, pp. 87

[14] M. Kanda, "Time-domain sensors and radiators", in Time-domain measurements in electromagnetic, E. K. Miller,Van Nostrand Reinhold Company Inc., 1986, pp. 125. 
[15] D. Ghosh, A. De, M. C. Taylor, T. K. Sarkar, M. C. Wicks and E. L. Mokole, "Transmission and reception by Ultra-Wideband (UWB) antennas “, IEEE Antennas and Propag. Mag, vol. 48, no. 5, pp. 67-99, Oct. 2006.

[16] K. Rambabu, A. E.-C. Tan, K. K.-M. Chan and M. Y. W. Chia, "Estimation of antenna effect on ultra-wideband pulse shape in transmission and reception", IEEE Trans. Electromagnetic Comp., vol. 51, no. 3, pp. 604-610, Aug. 2009.

[17] K. Siwiak, D. McKeown "Radiation of UWB signals", in Ultrawideband radio technology, Ed. John Wiley \& Sons Ltd, 2004, pp. 111.

[18] M. E. Bialkowski and A. M. Abbosh, "Design of a compact UWB outof-phase power divider", IEEE Microwave and Wireless Components Letters, vol. 17, no. 4, pp. 289-291, Apr. 2007.

[19] Y. Duroc, A. Ghiotto, T. P. Vuong and S. Tedjini, "UWB antennas: System with transfer function and impulse response", IEEE Trans. Antennas Propag., vol. 55, no. 5, pp. 1149-1451, May 2007.

[20] B. Scheers, M. Acheroy and A. Vander Vorst, "Time-domain simulation and characterisation of TEM horn using a normalised impulse response", in IEE Proc. Microwave Antenna and Propagation., vol. 147, no. 6, pp. 463-468, Dec. 2000.

[21] M. Bury, Y. Yashchyshyn, "Pulse response of UWB antenna: Meaning and Simple measurement procedure", in Proc. $2^{\text {nd }}$ European Conf. on Antenna and Propag., Edinburgh, 2007, pp.1-6.

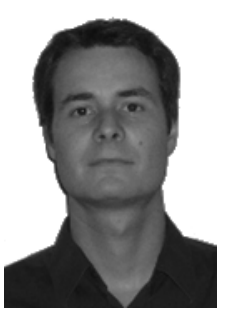

Antoine Dumoulin (S'11) received his honours degree in computer and communications engineering, from the Dublin Institute of Technology, Ireland in 2008. He is currently working towards the Ph.D. degree in microwave antenna engineering, with the Antenna and High Frequency Research Centre, School of Electronic and Communications Engineering, Dublin Institute of Technology.

His research interests include integrated UWB antennas, time domain characterization, signal processing, energy scavenging antennas, asset tag location systems and radar systems. He received a commercialization award in 2010 for technologies licensed to local and international companies.

Matthias John (M '07) received the degree (Dipl. Inf.) in computer science from the Deutsche Telekom University of Applied Science, Leipzig, Germany in 2004 and the Ph.D. in Microwave Antenna Engineering from the Dublin Institute of Technology, Dublin, Ireland in 2008.

He is currently working as a postdoctoral researcher in the Antenna and High Frequency Research Centre in the Dublin Institute of Technology. His research interests include UWB antennas, efficient numerical optimization techniques, antenna miniaturization, RFID and imaging applications. He is driving commercial activity in the center and received commercialization awards in 2009, 10 and 11 for technologies licensed to Irish and international companies. He also received a 2011 CST Award for work on "Miniature Ceramic Dual-PIFA Antenna to Support Band Group 1 UWB Functionality in Mobile Handset". He is part of the CTVR telecommunication research center and funded by Science Foundation Ireland.

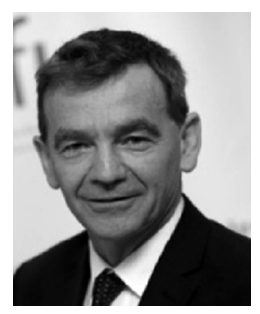

Max Ammann (M'96-SM'08) received the Council of Engineering Institution Part II degree in 1980 and the Ph.D. degree in microwave antenna design from Trinity College, University of Dublin, Dublin, Ireland, in 1997.

He is a Senior Lecturer in the School of Electronic and Communications Engineering, Dublin Institute of Technology, where he is the Director of the Antenna and High Frequency Research Centre. He also leads the antenna research within Ireland's Telecommunications Research Centre (CTVR), Dublin. He spent eight years on radio systems engineering and antenna design for TCL/Philips Radio Communications Systems, Dublin, where he was responsible for commissioning the Nationwide Communications Network for Ireland's national police force. In 1986 he joined the DIT as a Lecturer and was promoted to Senior Lecturer in 2003. His research interests include electromagnetic theory, antenna miniaturization for terminal and ultra wideband applications, microstrip antennas, metamaterials, antennas for medical devices and the integration with photovoltaic systems. He has in excess of 180 peer-reviewed papers published in journals and international conferences. He has served as an expert to industry on various antenna technologies in the communications, medical, aviation and electronic security sectors in Ireland and abroad. The roles have included design assessment, design solutions, technological strategy reporting and assessment of compliance with international standards on human exposure to electromagnetic energy. The industrial contacts also stem from several successful transfers of fundamental design research into applied solutions.

Dr. Ammann received the 2006 best poster award at the Loughborough Antennas and Propagation Conference, the 2009 SFI best paper award in the China Ireland International Conference in ICT and commercialization awards for work with DecaWave Ltd, Taoglas Ltd and Sequoia Smart Solutions. He also received a 2008 CST University Publication Award for work on a "Wideband Reconfigurable Rolled Planar Monopole Antenna" and a 2011 CST Award for work on "Miniature Ceramic Dual-PIFA Antenna to Support Band Group 1 UWB Functionality in Mobile Handset". He sits on the management committee of the EU COST Action IC1102, "Versatile, Integrated, and Signal-aware Technologies for Antennas (VISTA)". As a member of the IEEE International Committee for Electromagnetic Safety, he participated in the revision of the IEEE Std. C95.1, 2005 standard for Safety Levels with Respect to Human Exposure to Radio Frequency Electromagnetic Fields, $3 \mathrm{kHz}$ to $300 \mathrm{GHz}$. He is also a member of the URSI Committee for Communications and Radio Science within the Royal Irish Academy, with expertise in Commission K: Electromagnetics in Biology and Medicine. He chaired the IEEE APS Special Session on Antennas for UWB Wireless Communication Systems, Columbus, Ohio, 2003 and was chair for the Antennas and Propagation Track for the 65th IEEE VTC, Dublin 2007. He was the local chair for the October 2008 EU COST IC0603 workshop and meeting in Dublin.

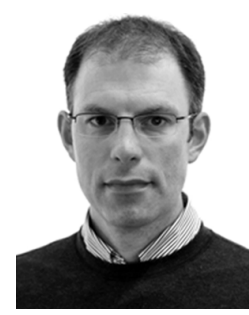

Patrick McEvoy (M'02) received the Dip.EE in telecommunications and electronic engineering from the Dublin Institute of Technology, Ireland in 1995, the M.Eng. degree in electronic communications engineering from the University of Hull, Hull, U.K. (with studies at L'Institut Supérieur d'Electronique de Paris, France) in 1998 and the Ph.D. degree in microwave antenna engineering from Loughborough University, Leicestershire, U.K., in 2007.

Currently, he is a senior researcher at the Antenna and High Frequency Research Centre, Dublin Institute of Technology (DIT), Dublin, Ireland. Prior to joining the DIT he was a Research Manager at the Centre for Mobile Communications Research, Loughborough University, where he worked on switched antennas for handheld terminals, applications of metamaterials, low specific absorption rate antenna design and antenna measurement systems. He has thirteen years of applied academic research and industrial experience that includes design, high-volume manufacturing and measurement systems for miniaturized microwave antennas. He is a corecipient of two awards for industrial commercialization of researched antenna technologies.

His main research interests include the electromagnetic interaction antenna-human tissue, hyperthermia applicators, microwave imaging, body area communications, ultra wideband antennas for frequency and time-domain applications and the integration of antennas with solar voltaic devices. He has published over 55 scientific papers and has helped to organize four international conferences on antennas and propagation. 